\title{
MENSURAÇÃO DA EROSÃO DA BORDA ANTERIOR DA GLENOIDE ATRAVÉS DO EXAME RADIOGRÁFICO: UMA FORMA SIMPLES DE REALIZAR A INCIDÊNCIA DE BERNAGEAU
}

\author{
ANTERIOR GLENOID RIM EROSION MEASURED BY X-RAY EXAM: \\ A SIMPLE WAY TO PERFORM THE BERNAGEAU PROFILE VIEW
}

Roberto Yukio Ikemoto', Luis Gustavo Prata Nascimento ${ }^{2}$, Rogerio Serpone Bueno ${ }^{3}$, Eric Strose ${ }^{3}$, Luis Henrique Oliveira Almeida ${ }^{3}$, Joel Murachovsky ${ }^{4}$

\section{RESUMO}

Objetivo: Analisar se a incidência radiográfica de Bernageau é adequada para estudar a borda anterior da glenoide e determinar a distância entre a borda anterior e posterior da glenoide. Método: Cinquenta pacientes (31 do sexo masculino) com idade média de 34 anos, foram avaliados posicionando-se o braço em flexão anterior de $160^{\circ}$ e o corpo a $70^{\circ}$ do chassi radiográfico, enquanto o posicionamento da ampola de raios-X era de $30^{\circ}$ crânio-caudal, centrada na espinha da escápula. Dos autores, três mensuraram três vezes a distância entre a borda anterior e posterior da glenoide. Foram estudadas a variabilidade e a reprodutibilidade dessa distância. Três cirurgiões de ombro realizaram uma avaliação subjetiva, respondendo se era possível a avaliação da borda anterior da glenoide na incidência estudada. Resultados: A distância foi em média 24,48mm \pm 0,332mm (esquerdo) e 24,82mm $\pm 0,316 \mathrm{~mm}$ (direito). O teste de AndersonDarling mostrou que as medidas tiveram distribuição normal e a correlação de Pearson's mostrou reprodutibilidade significativa ( $P$ $<0,01)$. O primeiro observador concluiu que $67 \%$ das radiografias eram adequadas para avaliar a borda anterior da glenoide, o segundo concluiu que $81 \%$ e o terceiro $78 \%$ eram satisfatórias para a avaliação. O coeficiente Kappa mostrou que o segundo e terceiro examinadores apresentaram concordância substancial em suas opiniões. Conclusão: A incidência de Bernageau proporciona uma radiografia adequada para o estudo da borda anterior da glenoide e para o cálculo da sua erosão após a comparação com o lado não acometido.

Descritores - Luxação do Ombro; Radiografia; Ortopedia; Planejamento

\begin{abstract}
Objective: To analyze whether the Bernageau view is adequate for studying the anterior glenoid rim and determining the distance between the posterior and anterior glenoid rim. Methods: Fifty patients (31 males) with a mean age of 34 years were evaluated by positioning their arm at $160^{\circ}$ forward flexion and body at $70^{\circ}$ with the $X$-Ray chassis, while positioning the $X$-ray tube at $30^{\circ}$ craniocaudal, centered on the scapula spine. Three of the authors measured the distance between the posterior and anterior glenoid rim three times. The variability and reproducibility of this distance were studied. Three shoulder surgeons performed a subjective evaluation by answering whether it was possible to evaluate the anterior glenoid rim in the view studied. Results: The average distance was 24.48 $\mathrm{mm} \pm 0.332 \mathrm{~mm}$ (left) and $24.82 \mathrm{~mm} \pm 0.316 \mathrm{~mm}$ (right). The Anderson-Darling test showed that this distance had a normal distribution and Pearson's correlation showed significant reproducibility $(P<0.01)$. The first observer found that $67 \%$ of the $X$-Rays images were suitable for evaluating the anterior glenoid rim. The second found that $81 \%$ were suitable and the third $78 \%$. The Kappa coefficient test showed that the second and third observers had substantial agreement of opinion. Conclusion: The Bernageau view provided a suitable X-Ray for studying the anterior glenoid rim and for assessing erosion after comparison with the unaffected side.
\end{abstract}

Keywords - Shoulder Dislocation; Radiography; Orthopedics; Planning

1 - Chefe do Grupo de Cirurgia do Ombro e Cotovelo da Faculdade de Medicina do ABC e do Hospital Ipiranga.

2 - Mestre em Medicina, Assistente do Grupo de Cirurgia do ombro e Cotovelo da Faculdade de Medicina do ABC e do Hospital Ipiranga.

3 - Pós-graduando em Ciências da Saúde, Assistente do Grupo de Cirurgia do ombro e Cotovelo da Faculdade de Medicina do ABC e do Hospital Ipiranga.

4 - Doutor em Medicina, Assistente do Grupo de Cirurgia do ombro e Cotovelo da Faculdade de Medicina do ABC e do Hospital Ipiranga.

Trabalho realizado no Grupo de Cirurgia do Ombro e Cotovelo da Faculdade de Medicina do ABC e Hospital do Ipiranga.

Correspondência: Rogerio Serpone Bueno - Rua Dráusio, 433 - Butantã - São Paulo, SP. CEP 05511-010 - E-mail: rsbueno@hotmail.com

Trabalho recebido para publicação: 26/10/09, aceito para publicação: 12/01/10. 


\section{INTRODUÇÃO}

A erosão óssea da borda anterior da glenoide tem sido relacionada a uma série de luxações do ombro e, dependendo do tamanho, pode haver a indicação do uso de enxerto ósseo para evitar a sua recorrência ${ }^{(1-4)}$.

Diversas incidências radiográficas foram descritas para estudar a borda anterior da glenoide e, consequentemente, a presença de erosão ${ }^{(2,5-7)}$. Apesar da radiografia ser um exame de baixo custo, poucos estudos demonstram que ela pode ser um exame adequado para medir a erosão da glenoide anteroinferior. Além disso, essas incidências dependem de equipamento especial para ajustar o posicionamento do paciente e são de difícil reprodução ${ }^{(2,5,6)}$.

Outros métodos para a avaliação da borda anterior da glenoide são a tomografia ${ }^{(6)}$, tomografia com reconstrução tridimensional $^{(8,9)}$ e na artroscopia do ombro com auxílio de "probe” milimetrado ${ }^{(10)}$. Entretanto, esses métodos são caros e, infelizmente, não estão disponíveis em todas as instituições.

O objetivo deste estudo é realizar uma análise prospectiva da borda anteroinferior da glenoide e, consequentemente, da presença de erosão com o posicionamento adequado dos sujeitos e uso de um aparelho comum de raios-X para reproduzir a incidência de Bernageau et $a l^{(5)}$. E também, analisar a distância entre as bordas anterior e posterior da glenoide, avaliando sua variabilidade e reprodutibilidade.

\section{MATERIAIS E MÉTODOS}

Durante os meses de janeiro e fevereiro de 2008, 50 adultos saudáveis, sem história prévia de patologia nos ombros foram submetidos a avaliação radiográfica de seus ombros (100 exames). A idade média dos indivíduos era de 34 anos (variando de 20 a 68 anos) e 31 eram do sexo masculino e 19 do feminino.

Este estudo foi aprovado pelo Comitê de Ética em Pesquisa de nossa instituição sob o número 082/2009. Todos os sujeitos leram e assinaram termo de consentimento informado antes de serem submetidos ao exame radiográfico.

\section{Posicionamento individual}

O estudo radiográfico foi realizado após a padronização do posicionamento dos sujeitos e da ampola de raios-X.

Cada indivíduo foi posicionado ficando em pé com o membro superior a ser estudado em flexão anterior de $160^{\circ}$ e o tórax em contato com o cassete radiográfico em um ângulo de $70^{\circ}$. Para assegurar a reprodutibilidade desse posicionamento, em todos os exames, uma almofada especialmente preparada era colocada na superfície anterior do tórax do sujeito examinado, assegurando que o tórax mantivesse essa angulação em relação ao cassete radiográfico (Figuras 1 e 2).

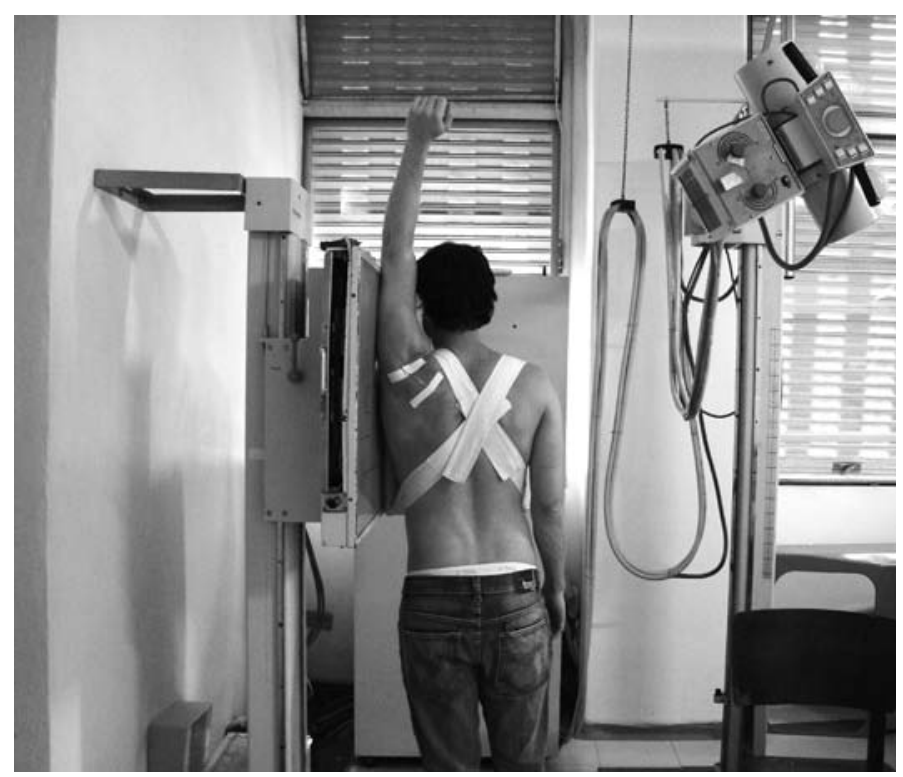

Figura 1 - Posicionamento do paciente mostrando a ampola de raios- $X$ em um ângulo de $30^{\circ}$ crânio-caudal e centrada na espinha da escápula, utilizando a almofada especial para corrigir a inclinação do tórax.

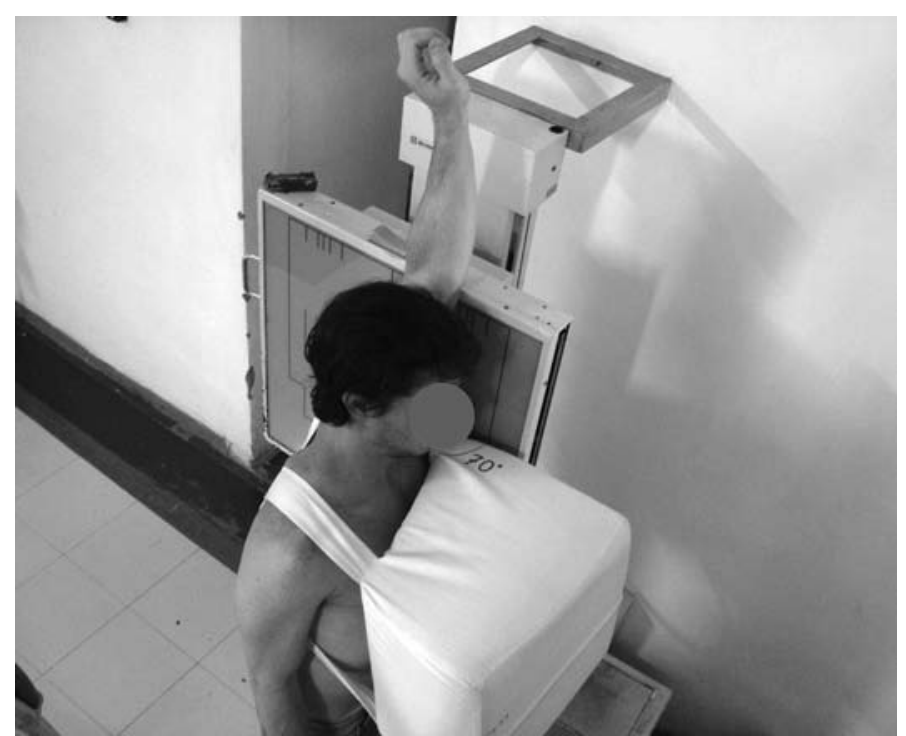

Figura 2 - Posicionamento do paciente com o tórax em ângulo $70^{\circ}$ com o chassi radiográfico (visão superior).

O aparelho radiográfico usado no estudo foi o Shimadzu 1/2P38D (Shimadzu Corporation Kyoto - Japan). A ampola de raios-X era posicionada a uma distância de $100 \mathrm{~cm}$ do ombro em estudo, em um ângulo de $30^{\circ}$ de inclinação caudal e centrada na espinha da escápula 
(Figura 1). A mesma técnica foi usada em todos os exames [65 kV ( $\pm 5 \mathrm{kV})$ e $20 \mathrm{~mA}$ ] e, o mesmo tipo e tamanho de filme radiográfico (FotoMed ${ }^{\mathrm{TM}}-24 \mathrm{x} 30 \mathrm{~cm}$ ).

Um pino de Steinmann número $3 \mathrm{com} 10 \mathrm{~cm}$ de comprimento foi colocado no ombro do sujeito em exame para correção da magnificação.

\section{Avaliação objetiva}

A avaliação objetiva foi realizada com a mensuração da distância entre as bordas anterior e posterior da glenoide (eixo anteroposterior), seguindo as corticais anterior e posterior da escápula.

Essa medida foi realizada por três examinadores independentes, cada examinador repetiu cada exame três vezes aleatoriamente sem saber qual caso estavam estudando.

\section{Avaliação subjetiva}

A avaliação subjetiva foi realizada por três cirurgiões de ombro e cotovelo convidados, que não participaram diretamente da elaboração do estudo. Eles responderam a um questionário, dando sua opinião se o posicionamento proposto era adequado para se realizar a incidência de Bernageau e, consequentemente, se era possível avaliar a borda anteroinferior da glenoide.

\section{Análise estatística}

$\mathrm{Na}$ análise estatística foi utilizado o programa SPSS (Statistical Package for Social Sciences, versão 13.0, SPSS Ind., Chicago - IL). Foi aplicado o coeficiente de correlação de Pearson para avaliar a reprodutibilidade intra e interobservador. $\mathrm{O}$ teste de normalidade de Anderson-Darling foi usado para avaliar se as medidas objetivas apresentavam distribuição normal e o coeficiente Kappa foi aplicado para avaliar a concordância entre as opiniões dos três cirurgiões de ombro e cotovelo. Consideramos como significante o $\mathrm{p}<0,05$.

\section{RESULTADOS}

\section{Análise objetiva}

A distância entre as bordas anterior e posterior da glenoide variou de $16,92 \mathrm{~mm}$ a $31,81 \mathrm{~mm}$ no ombro direito (média de 24,82mm $\pm 3,16 \mathrm{~mm}$ ) e, de 18,64mm a 32,22mm no ombro esquerdo (média de 24,48mm $\pm 3,32 \mathrm{~mm}$ ).

Foi avaliada a reprodutibilidade entre as três medidas realizadas por cada um dos três examinadores. Para isso, foi utilizado o teste de correlação de Pearson e observamos correlação significativa entre as três medidas de cada examinador, assim como entre os três examinadores, com $\mathrm{P}<0,05$ (Tabelas 1 e 2).

A distância entre a borda anterior e posterior da glenoide teve uma distribuição normal em 16 das 18 medidas realizadas neste estudo, utilizando-se o teste de normalidade de Anderson-Darling (95\% das amostras dentro do intervalo de confiança) (Figura 3).

\section{Análise subjetiva}

Os três especialistas em cirurgia do ombro e cotovelo foram denominados Observador 1, 2 e 3. Nas 100 avaliações realizadas pelo Observador 1 , ele acreditou que em $67 \%$ das vezes essa incidência radiográfica foi similar à incidência de Bernageau e, consequentemente, era adequada para a avaliação da borda anteroinferior da glenoide. O Observador 2 acreditou que em $81 \%$ dos casos as radiografias eram adequadas e o Observador 3 concluiu que $78 \%$ dos exames eram adequados para avaliação a borda anteroinferior da glenoide.

De acordo com teste do coeficiente de Kappa, observamos uma concordância significante entre as informações obtidas pelos observadores 2 e $3(\mathrm{P}=0,037)$. Entre os Observadores 1 e 2 e entre os Observadores 1 e 3 não houve correlação com significância estatística entre as medidas ( $p>0,05)$.

Tabela 1 - Distribuição das medidas realizadas pelos examinadores.

\begin{tabular}{|c|c|c|c|c|c|c|c|}
\hline \multirow[b]{2}{*}{ Pair of Variables } & \multirow[b]{2}{*}{$\mathrm{N}$} & \multicolumn{2}{|c|}{ Examinador 1} & \multicolumn{2}{|c|}{ Examinador 2} & \multicolumn{2}{|c|}{ Examinador 3} \\
\hline & & $\begin{array}{l}\text { Coeficiente de } \\
\text { correlação }\end{array}$ & Significância (p) & $\begin{array}{l}\text { Coeficiente de } \\
\text { correlação }\end{array}$ & Significância (p) & $\begin{array}{l}\text { Coeficiente de } \\
\text { correlação }\end{array}$ & Significância $(p)$ \\
\hline GLE-1-D x GLE-2-D & 50 & 0,976 & $<0,001$ & 0,949 & $<0,001$ & 0,962 & $<0,001$ \\
\hline GLE-1-D x GLE-3-D & 50 & 0,95 & $<0,001$ & 0,989 & $<0,001$ & 0,967 & $<0,001$ \\
\hline GLE-2-D x GLE-3-D & 50 & 0,942 & $<0,001$ & 0,952 & $<0,001$ & 0,962 & $<0,001$ \\
\hline GLE-1-E x GLE-2-E & 50 & 0,976 & $<0,001$ & 0,955 & $<0,001$ & 0,97 & $<0,001$ \\
\hline GLE-1-E x GLE-3-E & 50 & 0,978 & $<0,001$ & 0,586 & $<0,001$ & 0,974 & $<0,001$ \\
\hline GLE-2-E x GLE-3-E & 50 & 0,97 & $<0,001$ & 0,952 & $<0,001$ & 0,977 & $<0,001$ \\
\hline
\end{tabular}

Legenda: GLE1- primeira medida do examinador, GLE2- segunda medida do examinador, GLE3- terceira medida do examinador, D: lado direito e E: lado esquerdo. Fonte: Serviço de Radiologia. 
Tabela 2 - Distribuição comparativa das medidas realizadas pelos três examinadores no lado direito e no esquerdo.

\begin{tabular}{|c|c|c|c|c|c|}
\hline \multirow[b]{2}{*}{ Par de variáveis } & \multirow[b]{2}{*}{$\mathbf{N}$} & \multicolumn{2}{|c|}{ Lado direito } & \multicolumn{2}{|c|}{ Lado esquerdo } \\
\hline & & $\begin{array}{l}\text { Coeficiente de } \\
\text { correlação }\end{array}$ & Significância (p) & $\begin{array}{l}\text { Coeficiente de } \\
\text { correlação }\end{array}$ & Significância (p) \\
\hline Examinador1 GLE-1 x Examinador2 GLE-1 & 50 & 0,805 & $<0,001$ & 0,709 & $<0,001$ \\
\hline Examinador1 GLE-1 x Examinador3 GLE-1 & 50 & 0,782 & $<0,001$ & 0,77 & $<0,001$ \\
\hline Examinador2 GLE-1 x Examinador3 GLE-1 & 50 & 0,936 & $<0,001$ & 0,899 & $<0,001$ \\
\hline Examinador1 GLE-2 x Examinador2 GLE-2 & 50 & 0,759 & $<0,001$ & 0,634 & $<0,001$ \\
\hline Examinador1 GLE-2 x Examinador3 GLE-2 & 50 & 0,735 & $<0,001$ & 0,689 & $<0,001$ \\
\hline Examinador2 GLE-2 x Examinador3 GLE-2 & 50 & 0,877 & $<0,001$ & 0,814 & $<0,001$ \\
\hline Examinador1 GLE-3 x Examinador2 GLE-3 & 50 & 0,777 & $<0,001$ & 0,682 & $<0,001$ \\
\hline Examinador1 GLE-3 x Examinador3 GLE-3 & 50 & 0,719 & $<0,001$ & 0,723 & $<0,001$ \\
\hline Examinador2 GLE-3 x Examinador3 GLE-3 & 50 & 0,9 & $<0,001$ & 0,882 & $<0,001$ \\
\hline
\end{tabular}

Legenda: GLE1- Primeira medida do examinador, GLE2- Segunda medida do examinador, GLE3- Terceira medida do examinador.

Fonte: Serviço de Radiologia.

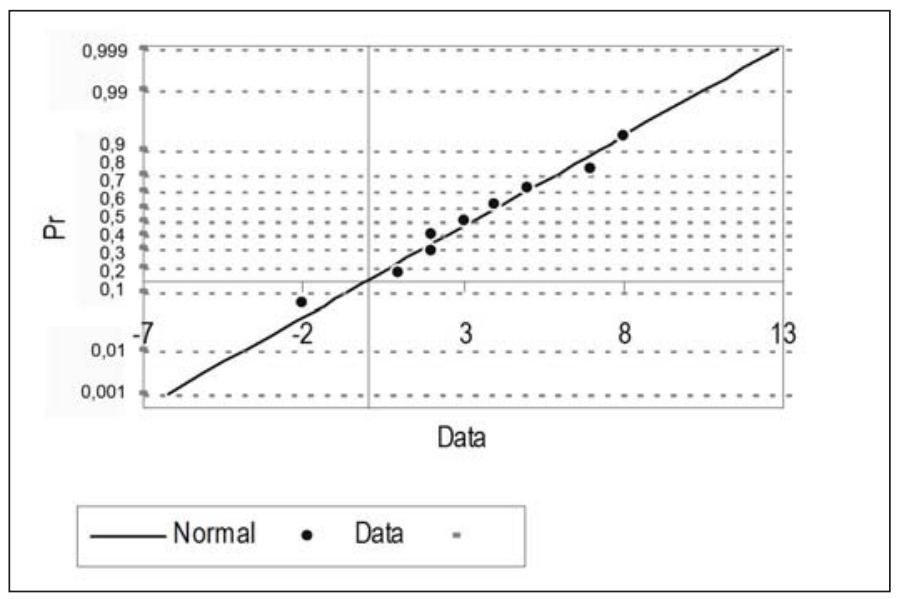

Figura 3 - Distribuição da primeira medida realizada pelo Examinador1 no ombro direito (exemplo de distribuição normal).

\section{DISCUSSÃO}

A presença de erosão da borda anteroinferior da glenoide pode levar a altas taxas de recorrência da luxação do ombro quando é realizado o tratamento convencional em uma luxação traumática, especialmente quando for maior que $25 \%^{(1,9,11,12)}$. Burkhart e De Beer et al ${ }^{(1)}$ apresentaram $67 \%$ de recidiva em casos com perda óssea significativa tratados artroscopicamente, enquanto a recorrência foi de apenas $4 \%$ nos casos sem erosão. Assim, o planejamento pré-operatório é de suma importância para evitar-se essa complicação.

Apesar de diversos autores demonstrarem que é possível determinar a presença de erosão significativa da glenoide durante o tratamento artroscópico (formato de pêra invertida) e mensurá-la com “probe” milimetrado ${ }^{(10)}$, outros observaram que a avaliação intraoperatória pode nem sempre estar correta ${ }^{(13)}$. Miyasaki et al demonstraram a dificuldade de determinar a presença de erosão da borda anteroinferior da glenoide, assim como de quantificá-la ${ }^{(14)}$. Entretanto, a importância de uma avaliação artroscópica prévia à cirurgia aberta está bem documentada $^{(4)}$.

A avaliação radiográfica é parte do planejamento pré-operatório, quando se tenta quantificar a presença de erosão óssea. As incidências de garth, axilar e west point foram citadas por Doneux et $a^{(15)}$ como as que poderiam ser solicitadas para estudar-se adequadamente a glenoide em casos de instabilidade anterior traumática do ombro; porém, nada é mencionado quanto à quantificação da possível perda óssea. Itoi et $a l^{(6)}$ demostraram que com a incidência de west point é possível quantificar a erosão da borda anteroinferior com boa precisão. Entretanto, para reproduzir essa incidência, é necessária a utilização de ampola radiográfica que angule $25^{\circ}$ em diferentes planos, sendo que algumas máquinas não possuem essa liberdade de movimentos. Além disso, no mesmo estudo, Itoi et $a l^{(6)}$ citam que essa incidência é de difícil reprodução nos pacientes, mesmo com aparelhos de raios-X que possuam essa amplitude de movimento.

Bernageau et $a l^{(5)}$ descreveram uma incidência radiográfica que possibilitava a avaliação de erosões ou fraturas da borda anteroinferior da glenoide; entretanto, a descrição de como reproduzi-la é incompleta. Edwards et $a l^{(2)}$, utilizaram esta incidência para avaliar lesões da borda anteroinferior da glenoide, posicionando o paciente com ajuda da fluoroscopia para assegurar a reprodutibilidade.

Em nosso estudo foi possível reproduzir a mesma incidência radiográfica utilizando um aparelho simples de raios-X sem o uso de fluoroscopia de suporte. Três especialistas em cirurgia do ombro, que não participaram diretamente do estudo, avaliaram 100 radiografias e ob- 
servaram que na maioria dos casos, foi possível se avaliar adequadamente a borda anteroinferior da glenoide.

A mensuração da distância proposta neste estudo provou-se reprodutível e com distribuição normal, sendo que os valores encontrados são similares aos achados em outros artigos ${ }^{(10,13)}$.

É importante ressaltar que não estamos propondo uma nova incidência radiográfica e, sim, descrevendo uma forma simples de reproduzir a incidência previamente descrita por Bernageau et al para avaliação da borda anteroinferior da glenoide (Figura 4). Uma vez sendo possível mensurar a distância entre a borda anterior e posterior da glenoide, pudemos avaliar a porcentagem de perda óssea, comparando a distância do ombro acometido com o saudável (Figura 5). Em nosso conhecimento, essa avaliação através de radiografias ainda não havia sido proposta até agora.

Em resumo, o exame radiográfico é mais barato e libera menos radiação que a tomografia computadorizada. Com isso, propomos que esse método de avaliação seja utilizado quando houver a suspeita clínica de erosão da borda anteroinferior da glenoide, como descrito por Bigliani et al $^{(16)}$, e realizar a tomografia computadorizada apenas nos pacientes em que a erosão já houver sido confirmada pela avaliação radiográfica.

\section{CONCLUSÃO}

Concluímos que esta incidência radiográfica é uma forma adequada e reprodutível de mensurar a presença de erosão da glenoide.

\section{REFERÊNCIAS}

1. Burkhart SS, De Beer JF. Traumatic glenohumeral bone defects and their relationship to failure of arthroscopic Bankart repairs: significance of the inverted-pear glenoid and the humeral engaging Hill-Sachs lesion. Arthroscopy. 2000;16(7):677-94.

2. Edwards TB, Boulahia A, Walch G. Radiographic analysis of bone defects in chronic anterior shoulder instability. Arthroscopy. 2003;19(7):732-9.

3. Boileau P, Villalba M, Hery JY, Balg F, Ahrens P, Neyton L. Risk factors for recurrence of shoulder instability after arthroscopic Bankart repair. J Bone Joint Surg Am. 2006;88(8):1755-63.

4. Speer KP, Deng X, Borrero S, Torzilli PA, Altchek DA, Warren RF. Biomechanical evaluation of a simulated Bankart lesion. J Bone Joint Surg Am. 1994;76(12):1819-26.

5. Bernageau J, Patte D, Debeyre J, Ferrane J. Value of the glenoid profil in recurrent luxations of the shoulder. Rev Chir Orthop Reparatrice Appar Mot. 1976;62(2 Suppl):142-7.

6. Itoi E, Lee SB, Amrami KK, Wenger DE, An KN. Quantitative assessment of classic anteroinferior bony Bankart lesions by radiography and computed tomography. Am J Sports Med. 2003;31(1):112-8.

7. Rockwood CA. X-ray evaluation of shoulder problems. The shoulder. Philadelphia: W.B. Saunders; 1990. p. 196-200.

8. Chuang TY, Adams CR, Burkhart SS. Use of preoperative three-dimensional computed tomography to quantify glenoid bone loss in shoulder instability.

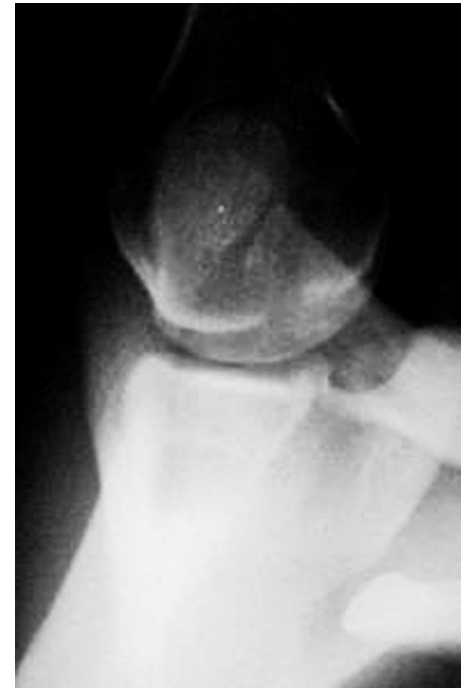

Fonte: Serviço de Radiologia

Figura 4 - Avaliação da borda anterior da glenoide.

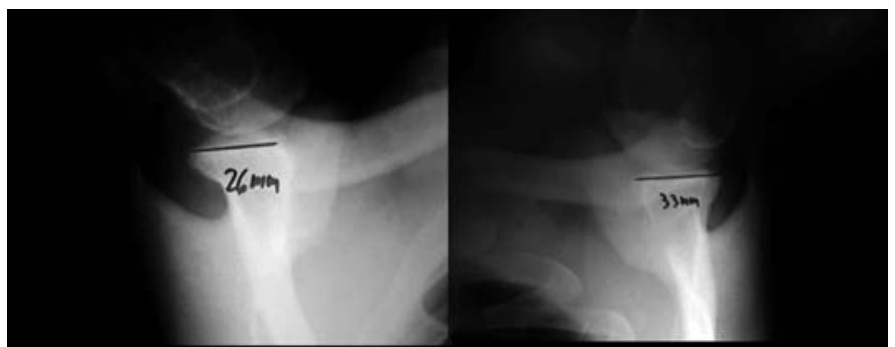

Figura 5 - Exemplo do cálculo da erosão, medindo-se a distância anteroposterior da borda da glenoide de ambos os ombros e comparando-as.

A distância média foi de $24,48 \mathrm{~mm} \pm 0,332 \mathrm{~mm}$ no ombro esquerdo e $24,82 \mathrm{~mm} \pm 0,316 \mathrm{~mm}$ no ombro direito.
Arthroscopy. 2008;24(4):376-82.

9. Sugaya H, Moriishi J, Dohi M, Kon Y, Tsuchiya A. Glenoid rim morphology in recurrent anterior glenohumeral instability. J Bone Joint Surg Am. 2003;85(5):878-84.

10. Burkhart SS, Debeer JF, Tehrany AM, Parten PM. Quantifying glenoid bone loss arthroscopically in shoulder instability. Arthroscopy. 2002;18(5):488-91.

11. Carr AJ. Biomechanics of shoulder stability. Curr Orthop. 1996;10:146-50.

12. Rowe CR, Patel D, Southmayd WW. The Bankart procedure: a long-term endresult study. J Bone Joint Surg Am. 1978;60(1):1-16.

13. Kralinger F, Aigner F, Longato S, Rieger M, Wambacher $M$. Is the bare spot a consistent landmark for shoulder arthroscopy? A study of 20 embalmed glenoids with 3-dimensional computed tomographic reconstruction. Arthroscopy. 2006;22(4):428-32.

14. Miyazaki AN, Doneux PS, Fregoneze M, Silva LA, Checcia SL. Avaliação da capacidade de mensuração visual da erosão em um modelo de gesso simulando a cavidade glenoidal na luxação recidivante anterior do ombro. . Rev Bras Ortop. 2002;37(9):403-7.

15. Doneux PS, Checchia SL, Miyazaki NA. Padronização do estudo radiográfico da cintura escapular. Rev Bras Ortop. 1998;33(11):883-8.

16. Bigliani LU, Newton PM, Steinmann SP, Connor PM, Mcllveen SJ. Glenoid rim lesions associated with recurrent anterior dislocation of the shoulder. Am J Sports Med. 1998;26(1):41-5. 